\title{
Oscillatory dynamos and their induction mechanisms
}

\author{
M. Schrinner ${ }^{1}$, L. Petitdemange ${ }^{2}$, and E. Dormy ${ }^{1}$ \\ 1 MAG (ENS/IPGP), LRA, École Normale Supérieure, 24 rue Lhomond, 75252 Paris Cedex 05, France \\ e-mail: martin@schrinner.eu \\ 2 Max-Planck-Institut für Astronomie, Königstuhl 17, 69117 Heidelberg, Germany \\ Received 20 December 2010 / Accepted 5 April 2011
}

\section{ABSTRACT}

\begin{abstract}
Context. Large-scale magnetic fields resulting from hydromagnetic dynamo action may differ substantially in their time dependence. Cyclic field variations, characteristic for the solar magnetic field, are often explained by an important $\Omega$-effect, i.e., by the stretching of field lines because of strong differential rotation.

Aims. The dynamo mechanism of a convective, oscillatory dynamo model is investigated.

Methods. We solve the MHD-equations for a conducting Boussinesq fluid in a rotating spherical shell. We computed the dynamo coefficients for the resulting oscillatory model with the help of the so-called test-field method. Subsequently, these coefficients were used in a mean-field calculation to explore the underlying dynamo mechanism.

Results. The oscillatory dynamo model we consider is an $\alpha^{2} \Omega$ one. Although the fairly strong differential rotation of this model influences the magnetic field, the $\Omega$-effect alone is not responsible for its cyclic time variation. If the $\Omega$-effect is suppressed, the resulting $\alpha^{2}$-dynamo remains oscillatory. Surprisingly, the corresponding $\alpha \Omega$-dynamo leads to a non-oscillatory magnetic field. Conclusions. The assumption of an $\alpha \Omega$-mechanism does not explain the occurrence of magnetic cycles satisfactorily.
\end{abstract}

Key words. dynamo - magnetohydrodynamics (MHD) - magnetic fields - Sun: dynamo

\section{Introduction}

The study of the solar cycle has motivated dynamo theory for many decades. Hence, the solar dynamo has become the prototype of oscillatory dynamos. However, its explanation is still controversial (Jones et al. 2010). Most solar dynamo models have been built on the assumption of an $\alpha \Omega$-dynamo mechanism (Ossendrijver 2003); that is, the poloidal field results from the interaction of helical turbulence with the toroidal field ( $\alpha$-effect) whereas the toroidal field is thought to originate in the shearing of poloidal field lines by strong differential rotation $(\Omega$-effect). This attempt is attractive for mainly two reasons:

- First, the existence of a strong shear layer at the bottom of the solar convection zone is observationally well established and the importance of a resulting $\Omega$-effect is non-controversial.

- Second, Parker's plane layer model (Parker 1955) and in particular mean-field electrodynamics (Steenbeck et al. 1966) provide a very elegant theoretical framework for this approach. Within the mean-field theory, attention is focused on large-scale, i.e. averaged fields, only, and the induction equation may be replaced by a mean-field dynamo equation (Krause \& Rädler 1980)

$\frac{\partial \overline{\boldsymbol{B}}}{\partial t}=\nabla \times(\boldsymbol{E}+\overline{\boldsymbol{V}} \times \overline{\boldsymbol{B}}-\eta \nabla \times \overline{\boldsymbol{B}})$,

in which $\overline{\boldsymbol{B}}$ and $\overline{\boldsymbol{V}}$ denote the average magnetic and the average velocity field, $\eta$ stands for the magnetic diffusivity and $\mathcal{E}$ is the mean electromotive force. Moreover, it is assumed that $\mathcal{E}$ is homogeneous in the mean magnetic field and may be replaced by a parameterisation in terms of $\overline{\boldsymbol{B}}$ and its first derivatives

$\mathcal{E}=\mathrm{a} \overline{\boldsymbol{B}}+\mathrm{b} \nabla \overline{\boldsymbol{B}}$
In Eq. (2), the so-called dynamo coefficients $a$ and $b$ are tensors of second and third rank, respectively, and depend only on the velocity field and the magnetic diffusivity. The traditional $\alpha$-effect implemented in a large number of solar dynamo models (e.g. Steenbeck \& Krause 1969; Roberts 1972; Roberts \& Stix 1972; Stix 1976; Ossendrijver 2003; Brandenburg \& Subramanian 2005; Chan et al. 2008) corresponds to the isotropic component of a in relation (2), while the $\Omega$-effect results from the $\phi$-component of the $\nabla \times(\overline{\boldsymbol{V}} \times \overline{\boldsymbol{B}})$ term in Eq. (1).

However, a strong differential rotation is not a necessary condition for oscillatory solutions of the dynamo Eq. (1), as has been demonstrated in several papers (see e.g. Rädler \& Bräuer 1987; Baryshnikova \& Shukurov 1987; Schubert \& Zhang 2000; Rüdiger et al. 2003; Stefani \& Gerbeth 2003). These papers presented models in which the toroidal field is likewise generated from the poloidal field by an $\alpha$-effect ( $\alpha^{2}$-models) and investigated necessary constraints on a, the boundary conditions for the magnetic field, and the geometry of the dynamo region to obtain oscillatory solutions of Eq. (1). Recently, oscillatory dynamo models have also been investigated by means of direct numerical simulations. Mitra et al. (2010) performed dynamo simulations in a wedge-shaped spherical shell with an applied forcing and demonstrated again the existence of oscillatory $\alpha^{2}$-dynamo models.

However, the success of mean-field models in reproducing solar-like variations of the magnetic field relies partly on the large number of free parameters, i.e. on the arbitrary determination of the dynamo coefficients a and b. An alternative approach is presented by Pétrélis et al. (2009). They construct amplitude equations guided from symmetry considerations and analyse polarity reversals and oscillatons of the magnetic field resulting from the interaction between two dynamo modes.

Self-consistent, global, convective dynamo models with cyclic magnetic field variations have been first reported by 
Gilman (1983) and Glatzmaier (1985). More recent examples have been published by Busse \& Simitev (2006), and Goudard \& Dormy (2008). Convective dynamo simulations with stressfree mechanical boundary conditions (Busse \& Simitev 2006) exhibit a strong and a weak field branch, depending on the initial conditions for the magnetic field. If the magnetic field is initially weak, stress free boundary conditions enable the development of a strong zonal flow carrying most of the kinetic energy. The magnetic field resulting from these dynamos is somewhat smallscaled, often of quadrupolar symmetry and weak. Oscillatory solutions of the induction equation are typical for this dynamo branch.

A transition from steady to oscillatory dynamos may also be governed by the width of the convection zone; Goudard \& Dormy (2008) found oscillatory models by decreasing the shell width. In this study, we follow their approach and analyse the dynamo mechanism for these oscillatory models. In particular, we address the question whether an $\Omega$-effect is responsible for the cyclic variation of the magnetic field. Different from previous work, we determine the dynamo coefficients $a$ and $b$ from direct numerical simulations with the help of the test-field method (Schrinner et al. 2005, 2007). The application of $a$ and $b$ in a mean-field calculation reveals their importance for the generation of the magnetic field.

\section{Dynamo calculations}

We consider a conducting Boussinesq fluid in a rotating spherical shell and solve the equations of magnetohydrodynamics for the velocity $\boldsymbol{v}$, magnetic field $\boldsymbol{B}$ and temperature $T$ as given by Goudard \& Dormy (2008) with the help of the code PaRoDy (Dormy et al. 1998, and further developments),

$$
\begin{gathered}
E\left(\frac{\partial \boldsymbol{v}}{\partial t}+\boldsymbol{v} \cdot \nabla \boldsymbol{v}-\nabla^{2} \boldsymbol{v}\right)+2 z \times \boldsymbol{v}+\nabla P= \\
R a \frac{\boldsymbol{r}}{r_{\mathrm{o}}} T+\frac{1}{P m}(\nabla \times \boldsymbol{B}) \times \boldsymbol{B}, \\
\frac{\partial \boldsymbol{B}}{\partial t}=\nabla \times(\boldsymbol{v} \times \boldsymbol{B})+\frac{1}{P m} \nabla^{2} \boldsymbol{B}, \\
\frac{\partial T}{\partial t}+(\boldsymbol{v} \cdot \nabla)\left(T+T_{\mathrm{s}}\right)=\frac{1}{P r} \nabla^{2} T .
\end{gathered}
$$

Governing parameters are the Ekman number $E=v / \Omega L^{2}$, the (modified) Rayleigh number $R a=\alpha_{\mathrm{T}} g_{0} \Delta T L / v \Omega$, the Prandtl number $\operatorname{Pr}=v / \kappa$ and the magnetic Prandtl number $P m=v / \eta$. In these expressions, $v$ denotes the kinematic viscosity, $\Omega$ the rotation rate, $L$ the shell width, $\alpha_{\mathrm{T}}$ the thermal expansion coefficient, $g_{0}$ is the gravitational acceleration at the outer boundary, $\Delta T$ stands for the temperature difference between the spherical boundaries, $\kappa$ is the thermal and $\eta=1 / \mu \sigma$ the magnetic diffusivity with the magnetic permeability $\mu$ and the electrical conductivity $\sigma$. Furthermore, the aspect ratio is defined as the ratio of the inner to the outer shell radius, $r_{\mathrm{i}} / r_{\mathrm{o}}$; it determines the shell width.

In our models, convection is driven by an imposed temperature gradient between the inner and the outer shell boundary, at which the temperature is fixed. The mechanical boundary conditions are no slip at the inner and stress free at the outer boundary. Moreover, the magnetic field is assumed to continue as a potential field outside the fluid shell.

Time-averaged dynamo coefficients for an axisymmetric mean magnetic field were determined from direct numerical simulations as described in detail by Schrinner et al. (2007) and as recently discussed for time-dependent dynamo models by Schrinner (2011). Some of them are displayed in Appendix A. In a second step, these coefficients were applied in a mean-field model based on Eq. (1) written as an eigenvalue problem,

$\sigma \overline{\boldsymbol{B}}=\nabla \times \mathrm{D} \overline{\boldsymbol{B}}$

Mean fields here are axisymmetric fields and the linear operator $D$ is defined as

$$
\mathrm{D} \overline{\boldsymbol{B}}=\overline{\boldsymbol{V}} \times \overline{\boldsymbol{B}}+\mathrm{a} \overline{\boldsymbol{B}}+\mathrm{b} \nabla \overline{\boldsymbol{B}}-\frac{1}{P m} \nabla \times \overline{\boldsymbol{B}} .
$$

The time evolution of each mode is determined by its eigenvalue $\sigma$ and proportional to exp $(\sigma t)$. For more details concerning the eigenvalue calculation, we refer to Schrinner et al. (2010b).

We also consider the evolution of a kinematically advanced magnetic field, $\boldsymbol{B}_{\mathrm{Tr}}$, governed by a second induction equation

$\frac{\partial \boldsymbol{B}_{\mathrm{Tr}}}{\partial t}=\nabla \times\left(\boldsymbol{v} \times \boldsymbol{B}_{\mathrm{Tr}}\right)+\frac{1}{P m} \nabla^{2} \boldsymbol{B}_{\mathrm{Tr}}$.

The tracer field $\boldsymbol{B}_{\mathrm{Tr}}$ experiences the self-consistent velocity field at each time step but does not contribute to the Lorentz force and is passive in this sense (see also Schrinner et al. 2010a). Its evolution will be compared with mean-field results originating likewise from a kinematic approach. Moreover, a kinematically advanced tracer field allows us to test for the influence of the $\Omega$-effect in direct numerical simulations. In a numerical experiment, we subtract the contribution of the $\Omega$-effect and the mean meridional flow in the equation for the tracer field,

$$
\frac{\partial \boldsymbol{B}_{\mathrm{Tr}}}{\partial t}=\nabla \times\left(\boldsymbol{v} \times \boldsymbol{B}_{\mathrm{Tr}}\right)+\frac{1}{P m} \nabla^{2} \boldsymbol{B}_{\mathrm{Tr}}-\nabla \times\left(\overline{\boldsymbol{V}} \times \overline{\boldsymbol{B}}_{\mathrm{Tr}}\right),
$$

and study in this way the outcome of a kinematic $\alpha^{2}$-dynamo.

\section{Results}

The model under consideration has been previously studied by Goudard \& Dormy (2008). It is defined by $E=10^{-3}, R a=$ $100\left(=2.8 R a_{\mathrm{c}}\right), P m=5, P r=1$ and an aspect ratio of 0.65 . Except for the stress-free mechanical boundary condition applied at $r=r_{\mathrm{o}}$ and an increased aspect ratio, the governing parameters are those of a fairly simple, quasi-steady benchmark dynamo (Christensen et al. 2001). However, Goudard \& Dormy (2008) report a transition from steady, dipolar to oscillatory models for these parameter values. Note that the model requires a very high angular resolution up to harmonic degree $l_{\text {max }}=112$.

Figure 1 displays the radial component of the velocity field at a given radial level. A typical columnar convection pattern is visible, even though the convection columns are noticeably disturbed by the influence of the curved boundaries and a strong zonal flow carrying about 50 percent of the kinetic energy. The magnetic Reynolds number based on the rms-velocity and the shell width, $R m=v_{\mathrm{rms}} L / \eta$, is about 90 . The flow is symmetric with respect to the equatorial plane and convection takes place only outside the inner core tangent cylinder.

The evolution of the magnetic field is cyclic. In Fig. 2 (top) we plot the contours of the azimuthally averaged radial magnetic field at the outer shell boundary varying with time in a socalled butterfly diagram. A dynamo wave migrates away from the equator until it reaches mid-latitudes where the inner core tangent cylinder intersects the outer shell boundary. The magnetic field looks very small-scaled and multipolar. This is confirmed by the magnetic energy spectrum which is essentially 


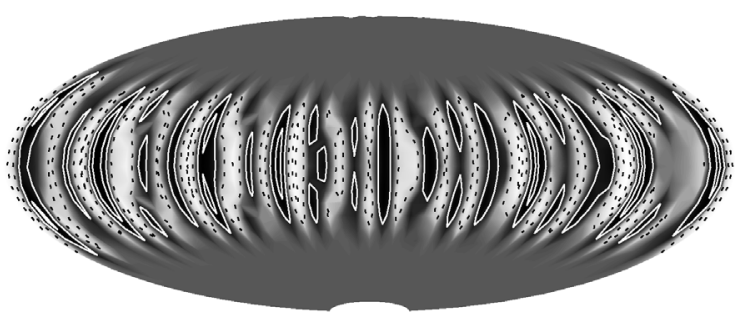

Fig. 1. Snapshot of the radial velocity of the considered dynamo model at $r=0.79 r_{0}$. The velocity component was normalised by its maximum absolute value, $\boldsymbol{v}_{r, \max }=24.46 \mathrm{v} / \mathrm{L}$. Hence, the colour-coding ranges from -1 , white, to +1 , black. Contour lines correspond to \pm 0.2 and \pm 0.6 .

white, except for a negligible dipole contribution. Furthermore, the magnetic field is weak, as expressed by an Elsasser number of $\Lambda=B_{\mathrm{rms}}^{2} /(\mu \rho \eta \Omega)=0.13$. This corresponds to a field strength of about $30 \%$ of its equipartition value $B_{\text {eq }}=\sqrt{E P m} v_{\text {rms }}$.

The kinematically advanced tracer field grows slowly in time, i.e., the model under consideration is kinematically unstable according to the classification by Schrinner et al. (2010a). But deviations of the tracer field from the actual field are hardly noticeable in the field morphology. Moreover, the very same dynamo wave persists in the kinematic calculation (see also Goudard \& Dormy 2008), as visible in Fig. 2 (middle). Note that the tracer field in Fig. 2 has evolved from random initial conditions.

A mean-field calculation based on the dynamo coefficients $\mathrm{a}, \mathrm{b}$ and the mean flow $\bar{V}$ determined from the selfconsistent model is presented in the bottom line of Fig. 2. The fastest growing eigenmodes form a conjugate complex pair and give rise to a dynamo wave that nicely compares with the direct numerical simulations. Because this model depends on the full a-tensor and the mean flow, we refer to it as an $\alpha^{2} \Omega$-dynamo.

The influence of the differential rotation may be suppressed in the kinematic calculation of the tracer field without changing any other component of the flow. A butterfly diagram resulting from a kinematically advanced field according to Eq. (9) is presented in Fig. 3 (top). The evolution of the magnetic field is again cyclic. Apart from small-scale variations on shorter time scales, a dynamo wave migrates from mid-latitudes towards the equator. This agrees with a corresponding mean-field calculation in which the mean flow $\overline{\boldsymbol{V}}$ in (7) has been canceled: the bottom chart of Fig. 3 provides the butterfly diagram stemming from the fastest growing eigenmodes of the resulting $\alpha^{2}$-dynamo. An explanation for the reason why direct numerical simulations and mean-field calculations compare somewhat better in Fig. 2 than in Fig. 3 is provided in Appendix B. The fairly regular oscillations at mid latitudes in the top panel of Fig. 3 are not captured by the time-averaged mean-field coefficients. Their origin is not well understood.

The time evolution of the related $\alpha \Omega$-dynamo is of further interest. Because the $\alpha$-effect is not directly accessible in direct numerical simulations, the corresponding $\alpha \Omega$-dynamo can only be realised in a mean-field calculation. In a first attempt, we set $\mathrm{a}_{r r}=\mathrm{a}_{\theta \theta}=0$ to suppress the generation of a toroidal field from the poloidal field by an $\alpha$-effect. Both components make major contributions to this process. The leading eigenmode resulting from this calculation is shown in Fig. 4; it is real, i.e., nonoscillatory, and close to marginal stability. The results remain similar if we neglect other, off-diagonal components of a.
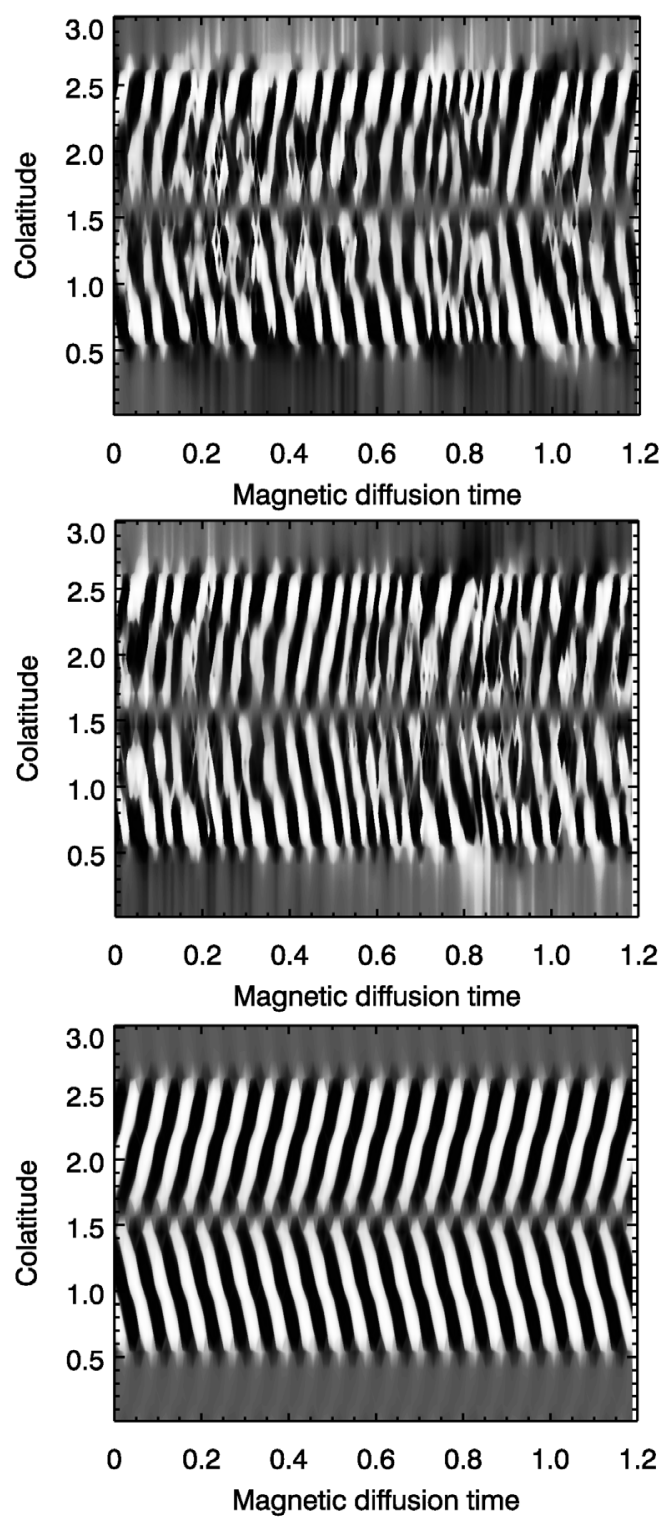

Fig. 2. Azimuthally averaged radial magnetic field at the outer shell boundary varying with time (butterfly diagram) resulting from a selfconsistent calculation (top), kinematic calculation according to (8) (middle) and mean-field calculation (bottom). The contour plots were normalised by their maximum absolute value at each time step considered. The colour-coding ranges from -1 , white, to +1 , black.

\section{Discussion}

The frequency and the propagation direction of the dynamo wave visible in Fig. 2 strongly depend on the differential rotation, in agreement with Busse \& Simitev (2006). We follow their approach and give an estimate for the cycle frequency by applying Parker's plane layer formalism (Parker 1955). To this end, we introduce a cartesian coordinate system $(x, y, z)$ corresponding to the $(\phi, \theta, r)$ directions and define mean quantities to be $x$ independent. Moreover, we write $\bar{B}=B \boldsymbol{e}_{x}+\boldsymbol{B}_{p}=B \boldsymbol{e}_{x}+\nabla \times A \boldsymbol{e}_{x}$ and reformulate (1) in the following simplified manner

$$
\begin{aligned}
& \frac{\partial A}{\partial t}=\alpha_{x x} B+\frac{1}{P m} \nabla^{2} A \\
& \frac{\partial B}{\partial t}=-\frac{\partial}{\partial y}\left(\alpha_{z z} \frac{\partial A}{\partial y}\right)+\frac{\mathrm{d} \overline{\boldsymbol{V}}_{x}}{\mathrm{~d} z} \frac{\partial A}{\partial y}+\frac{1}{P m} \nabla^{2} B
\end{aligned}
$$



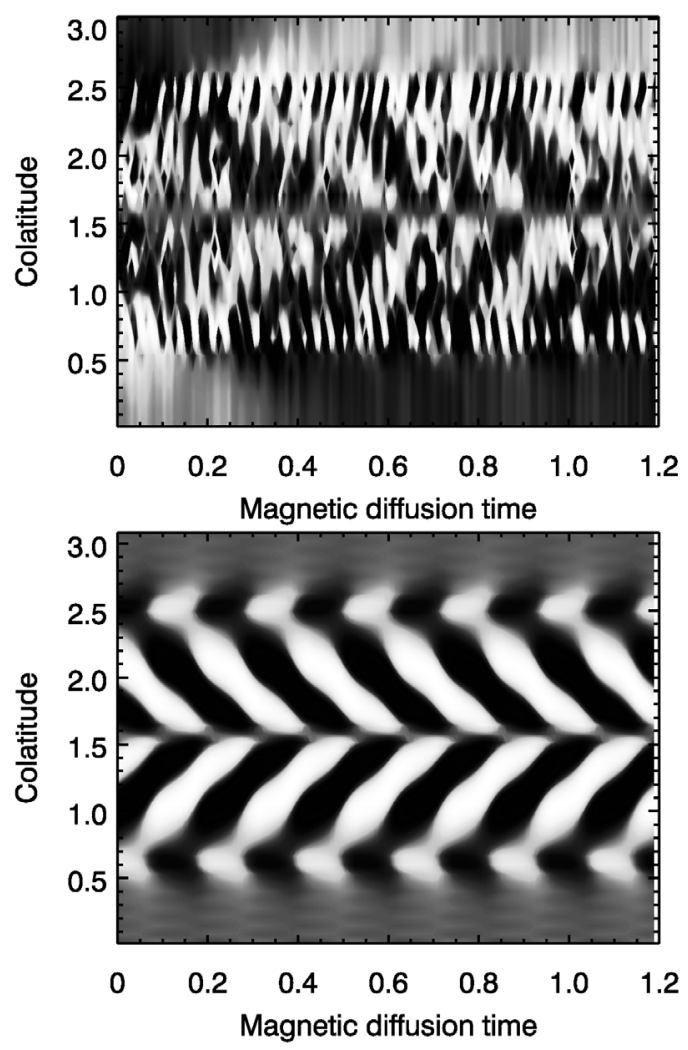

Fig. 3. Azimuthally averaged radial magnetic field at the outer shell boundary varying with time (butterfly diagram) resulting from a kinematic calculation with subtracted $\Omega$-effect (top) and a corresponding mean-field calculation (bottom). The contour plots are presented as in Fig. 2.

In the above equations, we considered only the dominant diagonal components of $\mathrm{a}, \mathrm{a}_{r r}$ and $\mathrm{a}_{\phi \phi}$ corresponding to $\alpha_{z z}$ and $\alpha_{x x}$; all components of $\mathrm{b}$ and the mean meridional flow were neglected. Furthermore, $\overline{\boldsymbol{V}}$ was assumed to depend only on $z$. Then, the ansatz

$$
(A, B)=(\hat{A}, \hat{B}) \exp \left(\mathrm{i}\left(\boldsymbol{k}_{y} y+\boldsymbol{k}_{z} z\right)+\sigma t\right)
$$

leads to

$$
\begin{aligned}
& p \hat{A}=\alpha_{x x} \hat{B}, \\
& p \hat{B}=\left(\alpha_{z z} \boldsymbol{k}_{y}^{2}+\mathrm{i} \boldsymbol{k}_{y}\left(\frac{\mathrm{d} \overline{\boldsymbol{V}}_{x}}{\mathrm{~d} z}-\frac{\partial \alpha_{z z}}{\partial y}\right)\right) \hat{A},
\end{aligned}
$$

with $p=\sigma+|\boldsymbol{k}|^{2} / P m$. From (13), (14), we derive a dispersion relation

$$
p^{2}=\alpha_{x x}\left(\alpha_{z z} \boldsymbol{k}_{y}^{2}+\mathrm{i} \boldsymbol{k}_{y}\left(\frac{\mathrm{d} \overline{\boldsymbol{V}}_{x}}{\mathrm{~d} z}-\frac{\partial \alpha_{z z}}{\partial y}\right)\right),
$$

from which real and imaginary parts of $\sigma$ can be calculated. If $\alpha_{x x}$ is positive (e.g. in the northern hemisphere), it follows

$$
\begin{aligned}
\lambda= & \Re(\sigma)=-|\boldsymbol{k}|^{2} / P m \\
& +\sqrt{\frac{\alpha_{x x}}{2}}\left(\sqrt{\left(\alpha_{z z} \boldsymbol{k}_{y}^{2}\right)^{2}+\left(\frac{\mathrm{d} \overline{\boldsymbol{V}}_{x}}{\mathrm{~d} z}-\frac{\partial \alpha_{z z}}{\partial y}\right)^{2} \boldsymbol{k}_{y}^{2}}+\alpha_{z z} \boldsymbol{k}_{y}^{2}\right)^{1 / 2}
\end{aligned}
$$

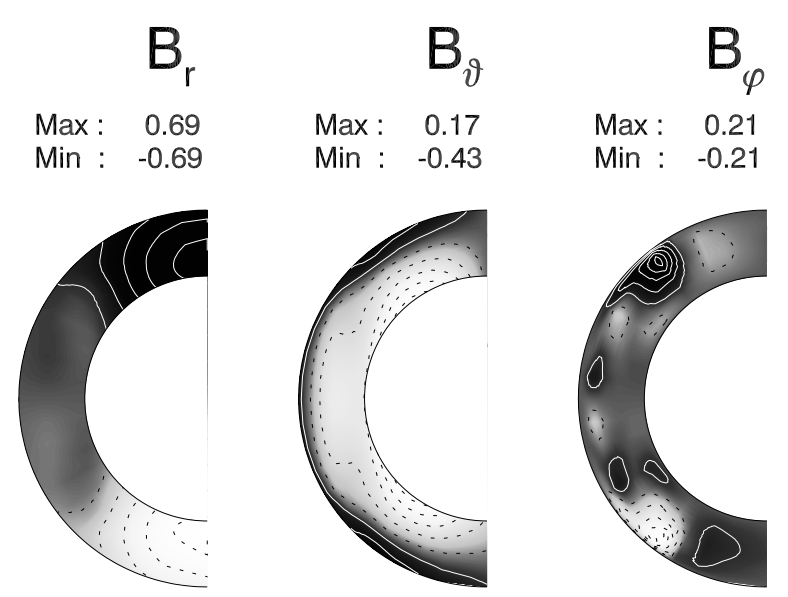

Fig. 4. Leading dipolar eigenmode resulting from a mean-field calculation with $a_{r r}=a_{\theta \theta}=0$. Contour plots of all three components are presented, each normalised separately by their maximum absolute values. Maxima and minima are written next to each plot. The colour-coding ranges from -1 , white, to +1 , black, and contour lines correspond to $\pm 0.1, \pm 0.3, \pm 0.5, \pm 0.7$ and \pm 0.9 .

and

$$
\begin{aligned}
\omega & =\mathfrak{J}(\sigma) \\
& = \pm \sqrt{\frac{\alpha_{x x}}{2}}\left(\sqrt{\left(\alpha_{z z} \boldsymbol{k}_{y}^{2}\right)^{2}+\left(\frac{\mathrm{d} \overline{\boldsymbol{V}}_{x}}{\mathrm{~d} z}-\frac{\partial \alpha_{z z}}{\partial y}\right)^{2} \boldsymbol{k}_{y}^{2}}-\alpha_{z z} \boldsymbol{k}_{y}^{2}\right)^{1 / 2} .
\end{aligned}
$$

The sign in (17) is determined by the sign of $\boldsymbol{k}_{y}\left(\mathrm{~d} \overline{\boldsymbol{V}}_{x} / \mathrm{d} z-\right.$ $\left.\partial \alpha_{z z} / \partial y\right)$. If we further assume that the frequency is dominated by differential rotation and neglect the $\alpha$-terms in (17), we estimate similar to Busse \& Simitev (2006)

$$
\omega=\mathfrak{J}(\sigma) \approx \pm\left(\frac{\pi}{L^{2}} \alpha_{x x} \sqrt{2 \bar{E}_{\mathrm{T}}}\right)^{1 / 2} .
$$

In (18), $\bar{E}_{\mathrm{T}}$ denotes the the kinetic energy density from the axisymmetric toroidal velocity field and $\boldsymbol{k}_{y} \approx 2 \pi / L$ was used. Approximating $\alpha_{x x}$ by the rms-value of $\mathrm{a}_{\phi \phi}, \alpha_{x x}=12 \mathrm{v} / \mathrm{L}$, and with $E_{\mathrm{T}}=71 v^{2} / L^{2}$, we find $\omega \approx \pm 106 \eta / L^{2}$ which is surprisingly close to $\omega= \pm 101 \eta / L^{2}$ in the full calculation presented in Fig. 2. Note that $\alpha_{x x}$ and $\mathrm{d} \overline{\boldsymbol{V}}_{x} / \mathrm{d} z$ are of the same order of magnitude and contribute equally to $\omega$. The sign in (18) is determined by the sign of the product $\mathrm{a}_{\phi \phi} \partial \overline{\boldsymbol{V}}_{\phi} / \partial r$, which is positive in the northern and negative in the southern hemisphere. Therefore, our estimate in (18) predicts a dynamo wave migrating away from the equator. This agrees with the simulations shown in Fig. 2.

However, the attempt to describe the model under consideration as an $\alpha \Omega$-dynamo fails. An oscillatory mode with a frequency close to the above estimate turns out to be clearly subcritical in a mean-field calcuation, if $\mathrm{a}_{r r}$ and $\mathrm{a}_{\theta \theta}$ are omitted. Instead, this model is governed by a real, dipolar mode close to marginal stability (see Fig. 4). Hence, the $\Omega$-effect is only partly responsible for the generation of the mean azimuthal field, as confirmed by Fig. 5. The middle panel compares the $\Omega$-effect, $r \overline{\boldsymbol{B}}_{r} \partial\left(r^{-1} \overline{\boldsymbol{V}}_{\phi}\right) / \partial r+r^{-1} \sin \theta \overline{\boldsymbol{B}}_{\theta} \partial\left(\sin \theta^{-1} \overline{\boldsymbol{V}}_{\phi}\right) / \partial \theta$ in greyscale with the mean azimuthal field displayed by superimposed contour lines. In particular, the elongated flux patches close to the inner core tangent cylinder are, if at all, negatively correlated with the $\Omega$-effect. Consistent with this finding, the poloidal axisymmetric magnetic energy density exceeds the toroidal one by $20 \%$. 

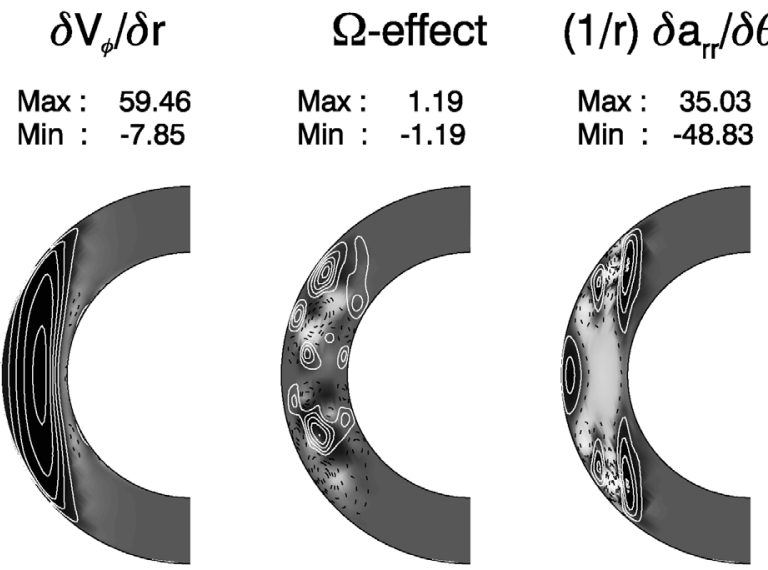

Fig. 5. Left: $\partial \overline{\boldsymbol{V}}_{\phi} / \partial r$ in units of $v / L^{2}$. Middle: $\Omega$-effect as given by $r \overline{\boldsymbol{B}}_{r} \partial\left(r^{-1} \overline{\boldsymbol{V}}_{\phi}\right) / \partial r+r^{-1} \sin \theta \overline{\boldsymbol{B}}_{\theta} \partial\left(\sin \theta^{-1} \overline{\boldsymbol{V}}_{\phi}\right) / \partial \theta$ (greyscale) and $\overline{\boldsymbol{B}}_{\phi}(\mathrm{su}-$ perimposed contour lines, solid [dashed] lines indicate positive [negative] values). Right: $(1 / r) \partial \mathrm{a}_{r r} / \partial \theta$ in units of $v / L^{2}$. Otherwise, the contour plots are presented in the same style as in Fig. 4.

Differential rotation alone is not responsible for the cyclic time evolution of the magnetic field, despite its influence on the frequency and the propagation direction of the dynamo wave. This is most clearly visible in Fig. 3. Simulations without differential rotation still lead to a dynamo wave even though its frequency and propagation direction have changed. In the framework of Parker's plane layer formalism, the frequency of this oscillatory $\alpha^{2}$-dynamo crucially depends on $-\partial \alpha_{z z} / \partial y$ instead of $\mathrm{d} \overline{\boldsymbol{V}}_{x} / \mathrm{d} z$. Note the additional minus sign, which might explain the reversed propagation direction if the assume that $\partial \alpha_{z z} / \partial y$ is predominantly positive. But different from the radial derivative of the mean azimuthal flow, $(1 / r) \partial \mathrm{a}_{r r} / \partial \theta$ is highly structured, changes sign in radial direction and exhibits localised patches of low negative values (see Fig. 5). Therefore, we do not attempt to give an estimate for the frequency similar to (18).

In order to better understand the influence of the mean flow on the frequency of the dynamo wave, we gradually changed the amplitude of $\overline{\boldsymbol{V}}$ in a series of kinematic calculations. Results are presented in Fig. 6. Stars denote frequencies obtained from eigenvalue calculations according to (6), whereas triangles stand for frequencies estimated from kinematic results from Eq. (8). In both cases, the amplitude of the mean flow $\overline{\boldsymbol{V}}$ was varied by multiplication with a scale factor $f$. For $f=1$, the original calculation is retained, while for $f=0$, we reproduce the $\alpha^{2}$-dynamo already discussed above. Frequencies of dynamo waves resulting from direct numerical simulations according to (8) were meassured for $f=1,0.7$, and 0.5 . Owing to the turbulence present in the simulations, these are very rough estimates and error bars have been included. Nevertheless, the results obtained satisfactorily agree with the eigenvalue calculations. The frequencies in Fig. 6 decrease continously with decreasing scale factors. If the amplitude of $\overline{\boldsymbol{V}}$ is reduced to 25 percent of its original value, $\omega$ changes sign and the propagation direction of the dynamo wave is reversed. The dashed-dotted line in Fig. 6 gives $\omega$ according to (18) as predicted for an $\alpha \omega$-dynamo. It matches the numerical results if $\mathrm{d} \overline{\boldsymbol{V}} / \mathrm{d} z$ dominates in (17) but clearly deviates for smaller amplitudes. On the other hand, it is illustrative to use relation (17) to model the dependence of $\omega$ on the mean flow. If we set $\partial \alpha_{z z} / \mathrm{d} y=0.25 \mathrm{~d} \overline{\boldsymbol{V}} / \mathrm{d} z$ and determine a representative value for $\alpha_{z z}$ inverting (17) for $\mathrm{d} \overline{\boldsymbol{V}} / \mathrm{d} z=0$ and $\omega=-29.15 \eta / L^{2}$, the dashed line in Fig. 6 results from (17). It fits the numerical data

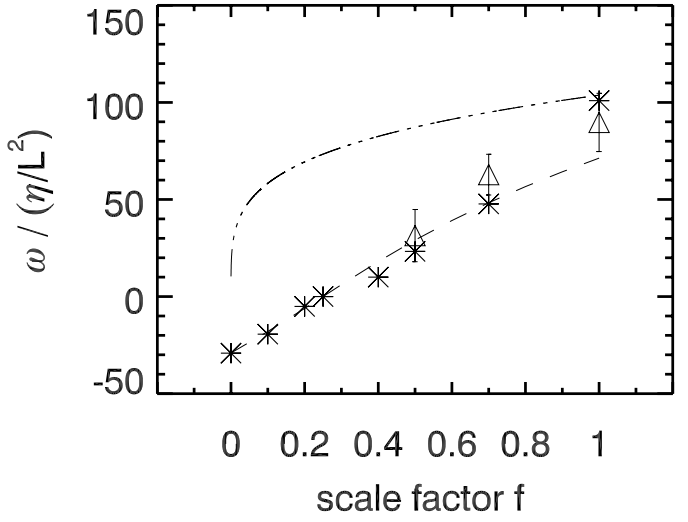

Fig. 6. Frequencies resulting from kinematic calculations in which the amplitude of the mean flow was changed by multiplication with a scale factor, $f$. Stars denote frequencies stemming from an eigenvalue calculation according to (6), whereas triangles are estimates obtained from kinematic results from (8). The dashed-dotted line gives frequencies as predicted for an $\alpha \omega$-dynamo by (18), while the dashed line represents $\omega$ as a function of $\overline{\boldsymbol{V}}$ for an $\alpha^{2} \omega$-dynamo according to (17).

quite well and converges towards the frequencies predicted for an $\alpha \omega$-dynamo if the amplitude of $\overline{\boldsymbol{V}}$ is sufficiently high.

Let us stress again that some caution is needed in applying the present mean-field analysis to non-linear direct numerical simulations, because the dynamo model considered here is kinematically unstable. Strictly speaking, our mean-field results are only relevant for the kinematically advanced tracer field. But because the model is close to dynamo onset and only weakly non-linear, we believe that our interpretation is also valid for the fully self-consistent field. This is in particular confirmed by the fairly good agreement of the three butterfly diagrams presented in Fig. 2.

\section{Conclusions}

A particular dynamo mechanism does not seem to be responsible for the occurrence of periodically time-dependent magnetic fields. It turns out that the influence of the large-scale radial shear (the $\Omega$-effect) is not necessary for cyclic field variations. Instead, the action of small-scale convection, represented by a spatially structured dynamo coefficient $\mathrm{a}_{r r}$, turns out to be essential. For the model presented here, small convective length scales are forced by a thin convection zone. Additional investigations are needed to assess whether our finding is representative for a wider class of oscillatory models.

Acknowledgements. M.S. is grateful for financial support from the ANR Magnet project. The computations were carried out at the French national computing center CINES.

\section{Appendix A: Dynamo coefficients}

Figure A.1 displays some of the dynamo coefficients derived from the oscillatory dynamo model considered here. Referring to the traditional representation of the mean electromotive force (e.g. Rädler 1980, 2000),

$$
\mathcal{E}=-\alpha \cdot \overline{\boldsymbol{B}}-\gamma \times \overline{\boldsymbol{B}}-\beta \cdot(\nabla \times \overline{\boldsymbol{B}})-\boldsymbol{\delta} \times(\nabla \times \overline{\boldsymbol{B}})-\kappa \cdot(\nabla \overline{\boldsymbol{B}})^{(\mathrm{sym})},
$$

the diagonal components of the $\alpha$-tensor and those of the the mean-field diffusivity tensor, $\mathrm{D}_{\kappa \lambda}=\eta \delta_{\kappa \lambda}+\beta_{\kappa \lambda}$, are presented. The weak negative contributions of $\mathrm{D}_{r r}$ are negligible in the 

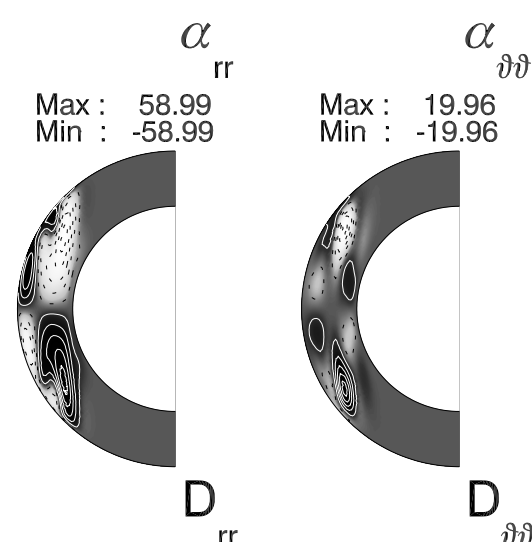

$\alpha_{\text {ขข }}$

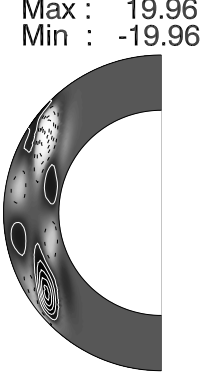

rr
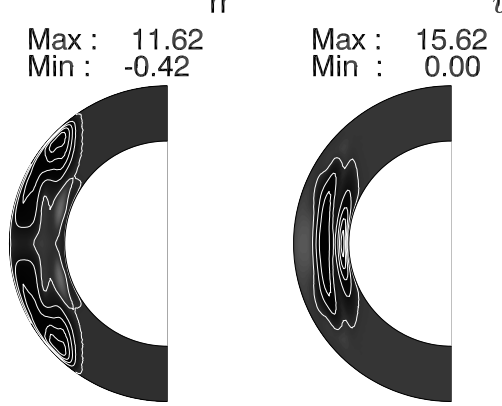

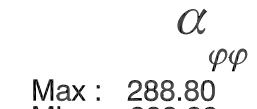

Min : -288.80

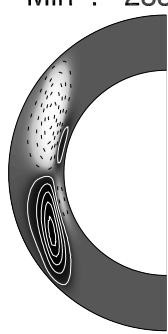

ขิ

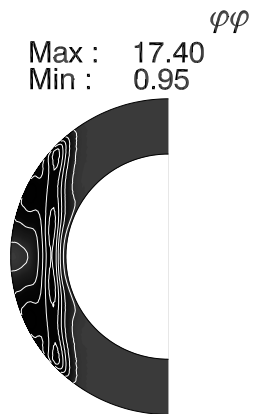

Fig. A.1. Contour plots of the diagonal components of the $\alpha$-tensor in units of $\eta / L$ and of the mean-field diffusivity tensor in units of $\eta$ for the oscillatory dynamo model. The contour plots are presented in the same style as in Fig. 4.

mean-field calculations. The dynamo coefficients $a$ and $b$ introduced in Eq. (2) are related to $\alpha$ and $\beta$ via

$\alpha_{\kappa \lambda}=-1 / 2\left(\mathrm{a}_{\kappa \lambda}+\mathrm{a}_{\lambda \kappa}\right)$

$\beta_{\kappa \lambda}=1 / 4\left(\epsilon_{\kappa \mu \nu} \mathrm{b}_{\lambda \mu \nu}+\epsilon_{\lambda \mu \nu} \mathrm{b}_{\kappa \mu \nu}\right)$.

\section{Appendix B: The use of time averaged-dynamo coefficients}

In the following, azimuthal averages are, as throughout in the paper, denoted by an overbar, time averages are expressed by brackets, $\langle\ldots\rangle$. Initially, dynamo coefficients were determined for an azimuthally averaged, mean magnetic field $\overline{\boldsymbol{B}}$. Hence, the evolution of the latter is given by

$$
\frac{\partial \overline{\boldsymbol{B}}}{\partial t}=\nabla \times\left(\mathrm{a} \overline{\boldsymbol{B}}+\mathrm{b} \nabla \overline{\boldsymbol{B}}+\overline{\boldsymbol{V}} \times \overline{\boldsymbol{B}}-\frac{1}{P m} \nabla \times \overline{\boldsymbol{B}}\right) .
$$

But the dynamo coefficients a, b and the mean flow $\bar{V}$ vary stochastically in time. In order to describe the average dynamo

action, we take in addition the time average of these quantities and write approximately

$$
\frac{\partial \overline{\boldsymbol{B}}}{\partial t} \approx \nabla \times\left(\langle\mathrm{a}\rangle \overline{\boldsymbol{B}}+\langle\mathrm{b}\rangle \nabla \overline{\boldsymbol{B}}+\langle\overline{\boldsymbol{V}}\rangle \times \overline{\boldsymbol{B}}-\frac{1}{P m} \nabla \times \overline{\boldsymbol{B}}\right) .
$$

We emphasise that there is no a priori relation between the left hand side and the right hand side of Eq. (B.2). The more a, b, and $\bar{V}$ fluctuate in time, the stronger the actual, azimuthally averaged magnetic field will deviate from our mean-field description. Among these three quantities, the mean flow $\bar{V}$ is almost time-independent, whereas a and $b$ vary considerably. This is the reason why the butterfly diagrams in Fig. 2 agrees better than in Fig. 3, where the stabilizing influence of the mean flow was omitted.

\section{References}

Baryshnikova, I., \& Shukurov, A. 1987, Astron. Nachr., 308, 89 Brandenburg, A., \& Subramanian, K. 2005, Phys. Rep., 417, 1

Busse, F. H., \& Simitev, R. D. 2006, Geophys. Astrophys. Fluid Dyn., 100, 341 Chan, K. H., Liao, X., \& Zhang, K. 2008, ApJ, 682, 1392

Christensen, U. R., Aubert, J., Cardin, P., et al. 2001, Phys. Earth Planet. Inter., 128,25

Dormy, E., Cardin, P., \& Jault, D. 1998, Earth Planet. Sci. Lett., 160, 15

Gilman, P. A. 1983, ApJS, 53, 243

Glatzmaier, G. A. 1985, ApJ, 291, 300

Goudard, L., \& Dormy, E. 2008, Europhys. Lett., 83, 59001

Jones, C. A., Thompson, M. J., \& Tobias, S. M. 2010, Space Sci. Rev., 152, 591 Krause, F., \& Rädler, K. 1980, Mean-field magnetohydrodynamics and dynamo theory, ed. L. J. Goodman, \& R. N. Love (Oxford: Pergamon Press Ltd.), 271

Mitra, D., Tavakol, R., Käpylä, P. J., \& Brandenburg, A. 2010, ApJ, 719, L1

Ossendrijver, M. 2003, A\&AR, 11, 287

Parker, E. N. 1955, ApJ, 122, 293

Pétrélis, F., Fauve, S., Dormy, E., \& Valet, J. 2009, Phys. Rev. Lett., 102, 144503

Rädler, K.-H. 1980, Astron. Nachr., 301, 101

Rädler, K.-H. 2000, in From the Sun to the Great Attractor, ed. D. Page, \& J. G. Hirsch (Berlin: Springer Verlag), Lect. Notes Phys., 556, 101

Rädler, K.-H., \& Bräuer, H. 1987, Astron. Nachr., 308, 101

Roberts, P. H. 1972, Phil. Trans. R Soc. Lond. A, 272, 663

Roberts, P. H., \& Stix, M. 1972, A\&A, 18, 453

Rüdiger, G., Elstner, D., \& Ossendrijver, M. 2003, A\&A, 406, 15

Schrinner, M. 2011, A\&A, submitted

Schrinner, M., Rädler, K., Schmitt, D., Rheinhardt, M., \& Christensen, U. 2005, Astron. Nachr., 326, 245

Schrinner, M., Rädler, K., Schmitt, D., Rheinhardt, M., \& Christensen, U. R. 2007, Geophys. Astrophys. Fluid Dyn., 101, 81

Schrinner, M., Schmitt, D., Cameron, R., \& Hoyng, P. 2010a, Geophys. J. Int., 182,675

Schrinner, M., Schmitt, D., Jiang, J., \& Hoyng, P. 2010b, A\&A, 519, A80

Schubert, G., \& Zhang, K. 2000, ApJ, 532, L149

Steenbeck, M., \& Krause, F. 1969, Astron. Nachr., 291, 49

Steenbeck, M., Krause, F., \& Rädler, K. 1966, Zeit. Nat. A, 21, 369

Stefani, F., \& Gerbeth, G. 2003, Phys. Rev. E, 67, 027302

Stix, M. 1976, A\&A, 47, 243 